\title{
Caracterização do processo de rigor mortis em músculos de eqüinos e maciez da carne
}

\author{
Caracterization of rigor mortis process of muscle horse and meat tenderness
}

\author{
Tatiana Pacheco Rodrigues ${ }^{1}$ Teófilo José Pimentel da Silva² ${ }^{\text {Eulógio Carlos Queiroz de Carvalho }}{ }^{3}$ \\ Mônica Queiroz de Freitas ${ }^{4}$ Flávia de Oliveira Paulino ${ }^{5}$
}

RESUMO

Esta pesquisa utilizou 12 eqüinos de diferentes idades, abatidos em um matadouro-frigorífico (SIF 1803) em Araguari-MG, e estudou a temperatura, $\mathrm{pH}$, comprimento de sarcômero em diferentes intervalos de tempo após abate $(1 \mathrm{~h}, 5 \mathrm{~h}$, $8 h, 10 h, 12 h, 15 h$ e 24h) e força de cisalhamento (maciez) dos músculos Longissimus dorsi e Semitendinosus, com intuito de caracterizar o desenvolvimento do processo de rigor mortis de eqüídeos durante o processamento industrial. A temperatura da câmara fria variou de $10,2^{\circ} \mathrm{C}$ a $4,0^{\circ} \mathrm{C}$ e a temperatura média inicial das carcaças foi de $35,32^{\circ} \mathrm{C}$ e a final de $4,15^{\circ} \mathrm{C} . \mathrm{O} \mathrm{pH}$ inicial médio do músculo Longissimus dorsi foi 6,49 e o final 5,63, e para o músculo Semitendinosus o $\mathrm{pH}$ inicial médio foi 6,44 e o final 5,70. A menor medida de sarcômero observada em

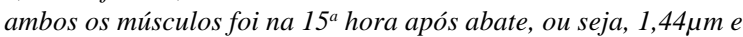
$1,41 \mu \mathrm{m}$, respectivamente. A carne dos equídeos adultos foi mais dura $(p<0,05)$ do que a dos jovens, bem como do músculo Semitendinosus quando comparado com o Longissimus dorsi.

Palavras - chave: carcaça eqüina, músculos, rigor mortis, maciez.

\section{ABSTRACT}

This work studied 12 horses at different ages butchered in a slaughterhouse in Minas Gerais State, Brazil (SIF 1803) and evaluated temperature, $\mathrm{pH}$, sarcomere length in different periods after slaughter ( $1 \mathrm{~h}, 5 \mathrm{~h}, 8 \mathrm{~h}, 10 \mathrm{~h}, 12 \mathrm{~h}, 15 \mathrm{~h}$, and 24 hours) as well as the shear force (meat tenderness) of the Longissimus dorsi and Semitendinosus muscles, aiming at characterizing the rigor mortis onset in the meat during industrial processing. The chilly room temperature varied from $10.2^{\circ} \mathrm{C}$ to $4.0^{\circ} \mathrm{C}$, and the mean initial carcass temperature was $35.32^{\circ} \mathrm{C}$ and the final one was $4.15^{\circ} \mathrm{C}$. The mean initial pH of Longissimus dorsi was 6.49 and the final one was 5.63; the mean initial pH of Semitendinosus was 6.44 and the final one was 5.70. The smallest sarcomere size obtained in both muscles occurred at 15 hours postmortem, and the sarcomere lengths were $1.44 \mu \mathrm{m}$ and $1.41 \mu \mathrm{m}$, respectively. The meat from adult horses was tougher than that from young ones ( $p<0.05)$, and the Semitendinosus muscle was tougher than Longissimus dorsi muscle.

Key words: horse carcass, muscle, rigor mortis, tenderness.

\section{INTRODUÇÃO}

No Brasil, a produção de carne de eqüinos afastados do trabalho e da reprodução tem por destino a exportação. $O$ preconceito contra a carne de eqüino torna o mercado interno inexpressivo, embora dispositivos legais permitam sua venda para consumo público. O consumo de carne de eqüídeos e seus derivados no Brasil são permitidos desde que conste nos rótulos a sua especificação, ou seja, o consumidor sabendo o que está comprando (TORRES \& JARDIM, 1985).

As características do processo de rigor mortis de carcaças de eqüídeos já foram estudadas em outros países, por autores como LAWRIE (1953), LEY (1996), WEYNERMANN \& DZAPO (1997). Estas

\footnotetext{
${ }^{1}$ Médico Veterinário, MSc., Professor Substituto do Departamento de Tecnologia de Alimentos, Universidade Federal Rural do Rio de Janeiro. E-mail: tatipr.br@bol.com.br.

${ }^{2}$ Médico Veterinário, PhD., Professor do Departamento de Tecnologia dos Alimentos, Universidade Federal Fluminense (UFF). E-mail: mtatjps@vm.uff.br.

${ }^{3}$ Médico Veterinário, DS., Professor de Patologia da UFF.

${ }^{4}$ Médico Veterinário, DS., Professor do Departamento de Tecnologia dos Alimentos, UFF.

${ }^{5}$ Médico Veterinário, Mestrando em Medicina Veterinária, UFF.
} 
características ainda não foram estabelecidas nas condições brasileiras.

O conhecimento a respeito das características do rigor mortis de carcaças de equiídeos nas condições brasileiras tem como modelo o fenômeno já observado em bovinos (ABREU, 1984), em frangos de corte (FREITAS, 1992), búfalos (SILVA, 1996) e em suínos (PINHEIRO et al., 2001).

A queda de $\mathrm{pH}$ e da temperatura durante o processo de rigor mortis das carcaças dos animais de açougue influenciam diretamente a qualidade da carne. A velocidade do rigor mortis é controlada, principalmente, pela reserva de glicogênio, $\mathrm{pH}$ e temperatura do músculo. Sendo assim, os objetivos desta pesquisa foram: caracterizar o comportamento do processo de rigor mortis nos músculos Longissimus dorsi e Semitendinosus em carcaças frigorificadas de eqüídeos; determinar o valor de $\mathrm{pH}$ e do comprimento de sarcômero dos músculos Longissimus dorsi e Semitendinosus, suas evoluções durante a retirada de calor sensível das carcaças na câmara de resfriamento logo após o abate e estabelecer a correlação desses valores com temperatura da carcaça e tempo após o abate; estudar o efeito da idade sobre a maciez da carne, nestes músculos.

\section{MATERIAL E MÉTODOS}

Foram escolhidos, ao acaso, 12 cavalos, sendo 06 jovens, com idades entre 03 e 3,5 anos, ou seja, menos de 06 dentes definitivos (03 machos e 03 fêmeas) e 06 adultos com idades entre 10 e 15 anos, com 06 ou mais dentes definitivos (03 machos e 03 fêmeas). Estes animais, procedentes de diversas regiões do Brasil (GO, TO, MG, SP e PR) foram transportados em caminhões próprios e, devido às características fisiológicas dos eqüinos, estes permaneceram 03 dias em repouso (recebendo alimentação), sob currais cobertos, sendo o jejum e a dieta hídrica realizados 24 horas antes do abate (do terceiro para o quarto dia). Os eqüídeos foram abatidos no matadouro-frigorífico Pomar Industrial Comercial - Araguari-MG. Após os procedimentos de abate e inspeção, as carcaças dos animais escolhidos (jovens com peso médio $116,33 \mathrm{~kg} \pm 16,77$ e adultos $165,02 \mathrm{~kg}$ $\pm 23,55$ ) foram estocadas em câmara frigorífica com temperatura média de $+1^{\circ} \mathrm{C}$ e umidade relativa média de $79 \%$. A temperatura da câmara foi aferida utilizando-se o termômetro digital de haste (modelo Gulterm 200) e a das meias-carcaças com utilização de termômetro digital Checktemp introduzindo-se sua haste metálica na profundidade de $05 \mathrm{~cm}$ na massa muscular na altura do ísquio, nos intervalos de 1h, 5h,
$8 \mathrm{~h}, 10 \mathrm{~h}, 12 \mathrm{~h}, 15 \mathrm{~h}$ e $24 \mathrm{~h}$ após o abate. A determinação do $\mathrm{pH}$ foi realizada de forma concomitante nos intervalos citados anteriormente, com potenciômetro Quimis ${ }^{\circledR}$ Q400, utilizando-se uma solução homogeneizada preparada com $10 \mathrm{~g}$ de amostra com $100 \mathrm{~mL}$ de água destilada, conforme SILVA et al. (1993).

De cada carcaça, foi feita a colheita de duas amostras, uma do músculo longo dorsal e outra do músculo semitendíneo. Fixaram-se garras metálicas duplas nos músculos e foram retiradas amostras no comprimento de $2,5 \mathrm{~cm}$, largura de $1,5 \mathrm{~cm}$ e espessura de $0,5 \mathrm{~cm}$, com auxílio de pinça e bisturi. As garras foram utilizadas com o intuito de manter o músculo nas mesmas condições em que se encontrava na carcaça, isto é, manter o comprimento de sarcômero inalterado até o momento do preparo dos cortes histológicos.

As amostras colhidas foram identificadas com o número da carcaça, músculo e intervalo de tempo após abate (1h, 5h, 8h, 10h, 12h, 15h e 24h). Logo após, as amostras foram colocadas em frascos plásticos de boca larga contendo fixador formalina tamponada a $10 \%(250 \mathrm{~mL})$. Os cortes histológicos foram corados com Hematoxilina Fosfotúngstica de Mallory, conforme BEHMER et al. (1976).

As lâminas foram lidas com auxílio de microscópio óptico de luz visível em objetiva de imersão, com utilização de óleo de cedro como óleo de imersão. Seguindo a técnica descrita por SLOSS \& KEMP (1978), o método consistiu na contagem de 10 sarcômeros de seis miofibrilas diferentes, com a medição do sarcômero sendo feita por régua de 10 micrômetros. A média obtida era multiplicada pelo fator de correção da objetiva de imersão (fator encontrado de $0,8 \mu \mathrm{m}$ ) e o valor expresso em micrômetros $(\mu \mathrm{m})$.

Para o teste de maciez, amostras de $250 \mathrm{~g}$ de cada músculo, de cada carcaça, após 24 horas de abate foram embaladas a vácuo, identificadas e transportadas em caixas isotérmicas para o Laboratório de Tecnologia de Carnes da Universidade Federal Fluminense, onde permaneceram estocadas $\mathrm{a} \pm 1^{\circ} \mathrm{C}$ até 96 horas após o abate. Realizou-se o cozimento destas até $70^{\circ} \mathrm{C}$ no centro geométrico da peça de carne (MORGAN et al., 1993) e após o resfriamento foram retirados 07 cilindros de $1,27 \mathrm{~cm}$ de diâmetro e comprimento de $7,0 \mathrm{~cm}$ que foram cisalhados uma só vez - ao meio pela máquina "Warner-Bratzler Meat Shear Force", para obtenção dos valores de força de cisalhamento, conforme KERTH et al. (1995). Os cortes utilizados foram o filé de lombo - músculo longo dorsal 
(músculo Longissimus dorsi); e o lagarto - músculo semitendíneo (músculo Semitendinosus).

O tratamento estatístico compreendeu análise de regressão para as variáveis dependentes pH (regressão linear), comprimento de sarcômero (regressão quadrática); e a variável independente intervalos de tempo após abate, com objetivo de estabelecer o modelo matemático que melhor interpretasse as variáveis e o comportamento delas em cada músculo, nos respectivos intervalos de tempo. Para o modelo de regressão escolhido $(p<0,05)$, testou-se o desvio da regressão $(p>0,15)$.

A análise de variância foi utilizada para comparar os valores médios de $\mathrm{pH}$, comprimento de sarcômero, maciez, temperatura das carcaças eqüinas, e para testar a significância das diferenças dos valores médios dos intervalos de tempo dos diferentes músculos após o abate ao nível de $5 \%$ ( $\mathrm{p}<0,05)$. Para comparação entre médias, utilizou-se o teste de Tukey.

Foi testada, ainda, a tendência de correlação entre as variáveis $\mathrm{pH}$, comprimento de sarcômero, temperatura da carcaça e tempo após o abate, determinando-se os respectivos coeficientes de correlação linear de Pearson. Para realizar as análises de variância e testar a correlação, foi utilizado o programa GRAPH PAD INSTAT e para realizar a análise de regressão foi utilizado o programa SAS (1985).

\section{RESULTADOS E DISCUSSÃO}

Os valores da temperatura da câmara fria, os valores médios da temperatura das carcaças, de
$\mathrm{pH}$ e comprimento de sarcômero se encontram na tabela 1. Os valores de $\mathrm{pH}$ obtidos no presente estudo concordam com as observações de LAWRIE (1953), que encontrou $\mathrm{pH}$ inicial de 6,95 $\pm 0,12 \mathrm{e}$ $\mathrm{pH}$ final de 5,51 $\pm 0,13$ no músculo longo dorsal de eqüídeos. Da mesma forma, WEYNERMMAN \& DZAPO (1997) estudaram as alterações post mortem em carcaças eqüinas e obtiveram $\mathrm{pH}$ inicial de 6,85 para o músculo longo dorsal e 6,87 para o músculo semitendíneo e pH final de 5,65 e 5,70 e concluíram que o sexo não influenciou significativamente o $\mathrm{pH}$. Entretanto o tempo post mortem influenciou o $\mathrm{pH}$ da carne, resultado este que é compatível com os dados desta pesquisa $(\mathrm{p}<0,05)$. O mesmo se pode dizer do trabalho de LEY (1996), que estudando as alterações post mortem em carcaças de 169 eqüinos e um asinino nos músculos adutor, longo dorsal e multifidus, verificou que idade, sexo, raça e transporte tiveram pouco efeito sobre as mudanças de $\mathrm{pH}$ e o músculo longo dorsal teve média de $6,881 \mathrm{~h}$ post mortem e média de $5,7924 \mathrm{~h}$ post mortem, médias que concordam com os trabalhos citados anteriormente.

Observou-se contração máxima em ambos os músculos na $15^{\text {a }}$ hora após abate, ou seja, o longo dorsal apresentando comprimento de sarcômero médio igual a $1,44 \mu \mathrm{m}$ e o semitendíneo $1,41 \mu \mathrm{m}$. Na literatura disponível não foi encontrado trabalho científico sobre o comprimento de sarcômero de músculos de eqüídeos. A figura 1 mostra fotomicrografias do músculo Longissimus dorsi durante o resfriamento industrial na $1^{\mathrm{a}}$ hora após abate, na $15^{\mathrm{a}}$ hora e na $24^{\mathrm{a}}$ hora.

Tabela 1 - Média (X) e desvio padrão (s) dos valores de temperatura $\left({ }^{\circ} \mathrm{C}\right)$ e $\mathrm{pH}$ de carcaças eqüinas, comprimento de sarcômero $(\mu)$ e temperatura da câmara frigorífica $\left({ }^{\circ} \mathrm{C}\right)$, nos diferentes tempos (h) após o abate, no Frigorífico Pomar, Araguari, MG, 2001.

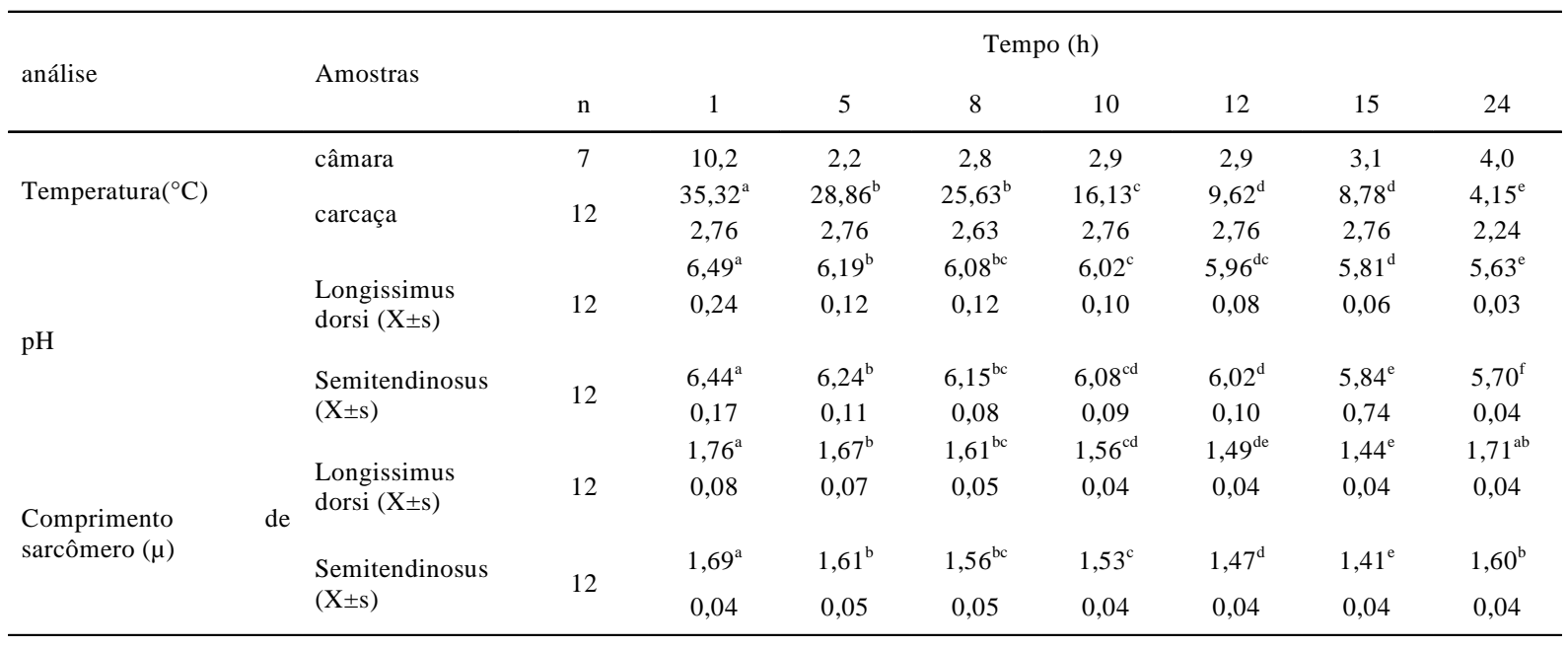

a, b, c, d, e, f Médias na mesma linha seguidas de diferentes letras diferem significativamente $(\mathrm{p}<0,05)$ 


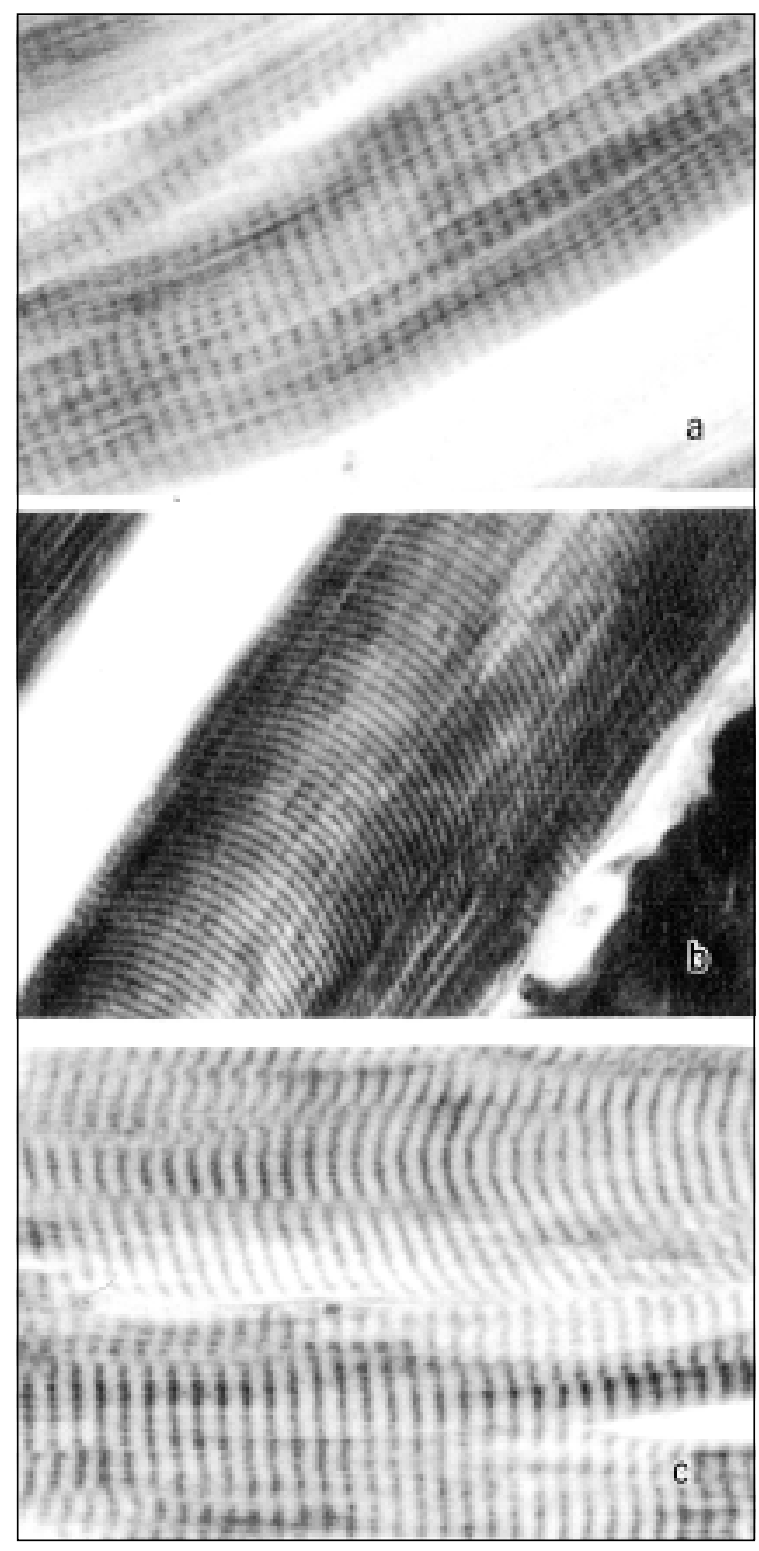

Figura 1 - Fotomicrografias do músculo Longissimus dorsi: durante o resfriamento industrial, mostrando as bandas A e I (aumento de 1000 vezes), fixado com formalina tamponada neutra e corado com Hematoxilina Fosfotúngstica de Mallory: a) na $1^{\underline{a}}$ hora após abate b) na $15^{\mathrm{a}}$ hora e c) na $24^{\mathrm{a}}$ hora.

Os resultados de força de cisalhamento (Tabela 2) foram de $1,87 \mathrm{~kg} \pm 0,19$ para o músculo longo dorsal nos animais jovens e $2,39 \mathrm{~kg} \pm 0,20$ nos animais adultos, e de $2,67 \mathrm{~kg} \pm 0,25$ para o músculo semitendíneo nos jovens e 4,04kg $\pm 0,65$ nos adultos. Esses resultados podem ser considerados como carnes macias, pois se encontram com força de cisalhamento abaixo de 6,0kg (SHACKLEFORD et al., 1997).
Tabela 2 - Média (X) e desvio padrão (s) dos valores de força de cisalhamento dos diferentes cortes (filé de lombo e lagarto), cisalhados 96 horas após o abate, de 12 equiinos abatidos no Frigorífico Pomar, Araguari, MG, 2001.

\begin{tabular}{llllll}
\hline \multirow{2}{*}{ Amostra } & \multicolumn{3}{l}{ Valores de força de cisalhamento $(\mathrm{Kg})$} \\
\cline { 2 - 6 } & $\mathrm{n}$ & \multicolumn{3}{c}{ Jovens } & \multicolumn{3}{c}{ Adultos } \\
\cline { 2 - 6 } & & $\mathrm{X}$ & $\mathrm{s}$ & $\mathrm{X}$ & $\mathrm{s}$ \\
\hline Filé de lombo & 42 & $1,87^{\mathrm{aA}}$ & $\pm 0,19$ & $2,39^{\mathrm{bA}}$ & $\pm 0,20$ \\
Lagarto & 42 & $2,67^{\mathrm{aB}}$ & $\pm 0,25$ & $4,04^{\mathrm{bB}}$ & $\pm 0,65$ \\
\hline
\end{tabular}

a, b, Médias na mesma linha seguidas de diferentes letras diferem significativamente $(\mathrm{p}<0,05)$.

${ }_{\mathrm{A}, \mathrm{B}}$ Médias na mesma coluna seguidas de diferentes letras diferem significativamente $(\mathrm{p}<0,05)$.

Os testes de correlação demonstraram alta correlação linear inversa entre temperatura da carcaça e tempo após abate $(\mathrm{r}=-0,92516)$ e alta correlação inversa entre $\mathrm{pH}$ e tempo após abate para os músculos longo dorsal e semitendíneo ( $\mathrm{r}=$ $-0,96784$ e $r=-0,97852$, respectivamente). Observouse, ainda, baixa correlação inversa entre tempo e comprimento de sarcômero dos músculos longo dorsal e semitendíneo ( $r=-0,2196$ e r= -0,3856); correlação linear baixa entre $\mathrm{pH}$ e comprimento de sarcômero do músculo longo dorsal $(\mathrm{r}=0,3657)$ e média entre $\mathrm{pH}$ e comprimento de sarcômero do músculo semitendíneo $(\mathrm{r}=0,4809)$. Observou-se, também, correlação média entre temperatura das carcaças e comprimento de sarcômero para os músculos longo dorsal e semitendíneo $(r=0,4465$ e $r=0,6016)$.

Os valores médios obtidos em cada tempo após abate estão representados em modelos gráficos juntamente com os modelos de regressão, coeficientes de determinação $\left(\mathrm{R}^{2}\right)$ e níveis de probabilidade $(\mathrm{p})$ (Figuras 2 e 3 ).

\section{CONCLUSÕES}

As quedas de temperatura e do $\mathrm{pH}$ acompanharam o desenvolvimento do processo de rigor mortis em ambos os músculos estudados até 24 horas após abate, seguindo os padrões normais da conversão do músculo em carne. E a contração máxima durante o processo de rigor mortis ocorreu em ambos os músculos na $15^{\mathrm{a}}$ hora após abate.

A carne dos cavalos adultos foi mais dura do que a dos mais jovens, e o filé de lombo (músculo Longissimus dorsi) foi mais macio do que o lagarto (músculo Semitendinosus), demonstrando que a idade e tipo de músculo influenciam na maciez da carne eqüina. 


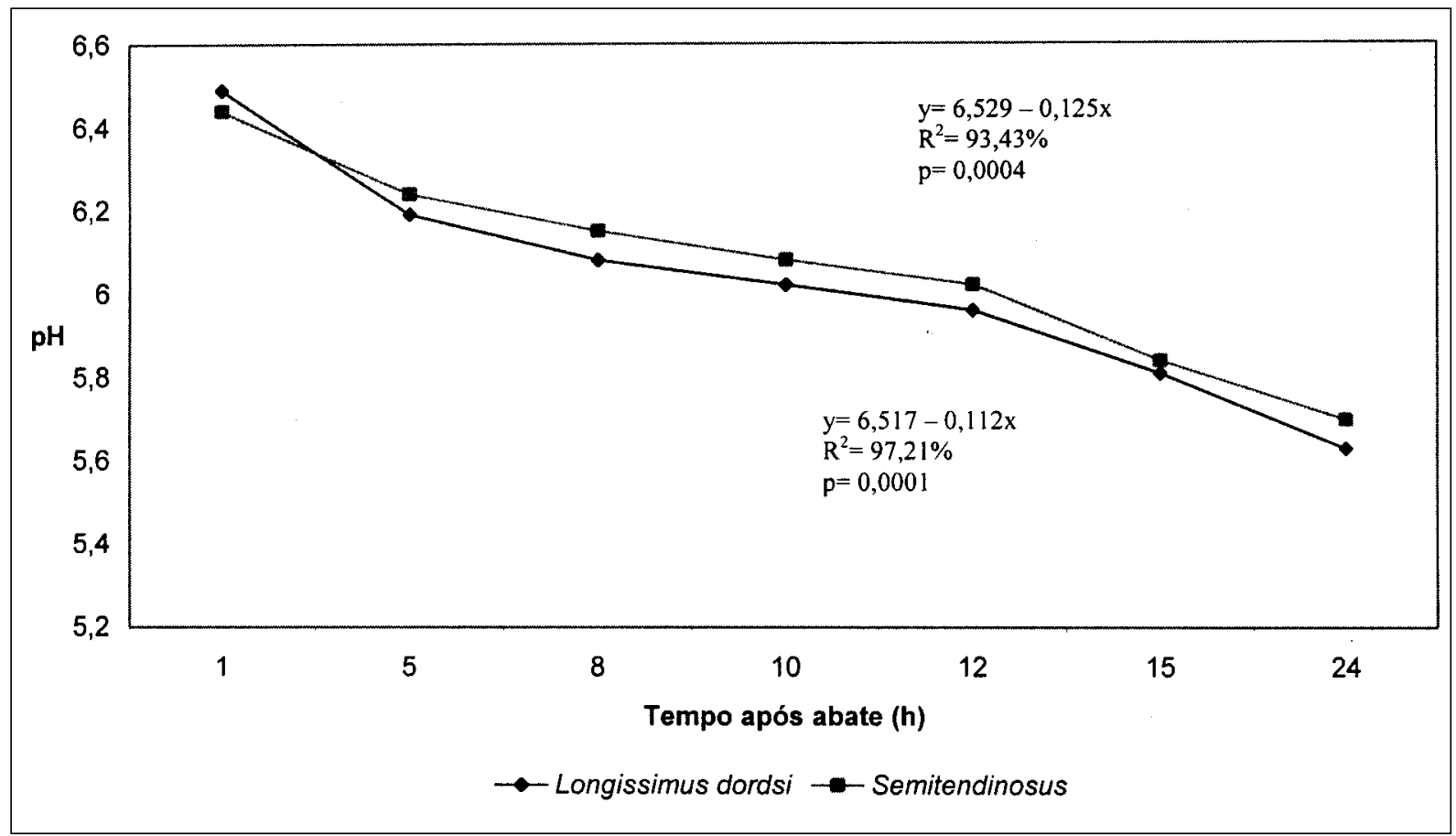

Figura 2 - Variação dos valores médios de pH dos músculos Longissimus dorsi e Semitendinosus de 12 carcaças eqüinas, nos diferentes tempos após abate, durante o resfriamento industrial no Frigorífico Pomar, Araguari, MG, 2001.

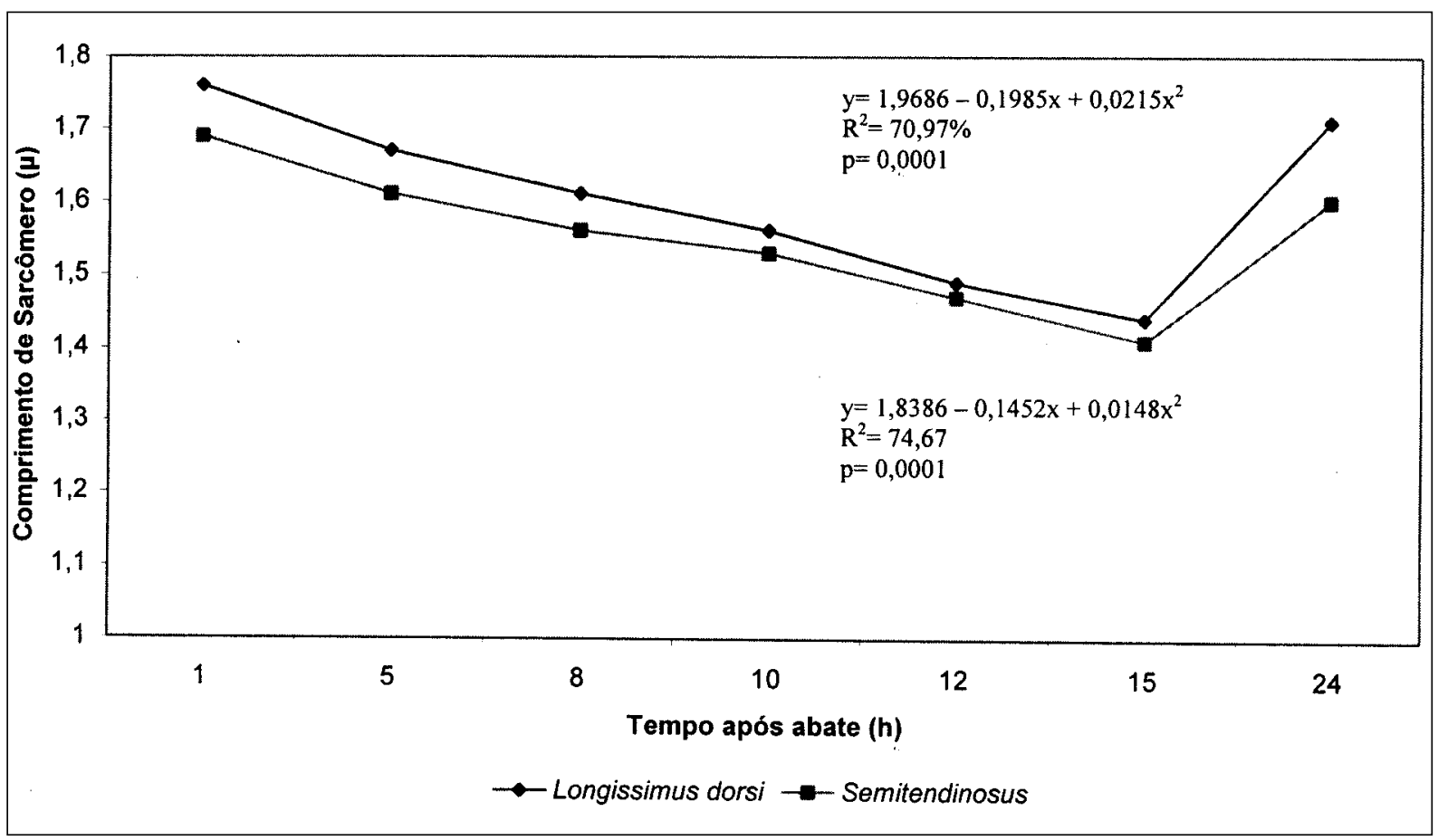

Figura 3 - Variação dos valores médios do comprimento de sarcômero dos músculos Longissimus dorsi e Semitendinosus de 12 carcaças eqüinas, nos diferentes tempos após abate, nas diferentes faixas etárias, durante o resfriamento industrial no Frigorífico Pomar, Araguari, MG, 2001.

Ciência Rural, v.34, n.4, jul-ago, 2004. 


\section{REFERÊNCIAS BIBLIOGRÁFICAS}

ABREU, R.L. Correlação entre tempo, temperatura e pH com comprimento de sarcômero no rigor mortis em carcaça frigorificada de bovino. 1984. 81f. Dissertação (Mestrado de Higiene Veterinária e Processamento Tecnológico de Produtos de Origem Animal) - Universidade Federal Fluminense.

BEHMER, O.A; TOLOSA, E.M.C.; NETO, A.G.F. Manual de técnicas para histologia normal e patológica. São Paulo : EDART - USP, 1976. 239p.

FREITAS, M.Q. Correlação entre pH e comprimento de sarcômero em músculos de aves (Gallus domesticus) durante o processamento industrial para obtenção de carcaças resfriadas. 1992. 68f. Dissertação (Mestrado de Higiene Veterinária e Processamento Tecnológico de Produtos de Origem Animal) Universidade Federal Fluminense.

KERTH, C.R.; MILLER, M.F.; RANSEY, C.B. Improvement of beef tenderness and quality traits with calcium chloride injection in beef loins 48 hours post mortem. Journal of Food Science, v.73, p.750-756, 1995.

LAWRIE, R.A. Onset of rigor mortis in various muscles of draught horse. Journal of Physiology, n.121, p.275-288, 1953.

LEY, T. Investigations of post-mortal changes in carcass of horses. Fleischwirtschafl, v.76, n.2, p.172-175, 1996

MORGAN, J. B. et al. Meat tenderness and the calpain proteolytic system in Longissimus muscle of young bulls and steers. Journal of Animal Science, v.71, n.6, p.1471-1476, 1993.
PINHEIRO, R.M.K.; SILVA, T.J.P.; CARVALHO, E.C.Q. Temperatura, $\mathrm{pH}$ e comprimento de sarcômero durante o rigor mortis em carcaça frigorificada de suínos abatidos em São Gonçalo-RJ. Higiene Alimentar, v.15, n.82, p.39-45, 2001 .

SAS Institute. SAS® User's guide statistics. Cary, NC : SAS Institute, 1985. 959p.

SILVA, T.J.P. et al. Effect of heating rate on shortening, ultraestruture and fracture behavior of prerigor beef muscle. Meat Science, v.33, n.1, p.1-27, 1993.

SILVA, F.E.R. Variação de pH e do comprimento de sarcômero em músculos de búfalos (Bubalus bubalis var. bubalis) durante o processamento industrial para obtenção de carcaças resfriadas. 1996. 69f. Dissertação (Mestrado de Higiene Veterinária e Processamento Tecnológico de Produtos de Origem Animal) - Universidade Federal Fluminense.

SHACKLEFORD, S.D.; WHEELER, T.L.; KOOHMARAIE, M. Tenderness classification of beef: Evaluation of beef Longissimus shear force a 1 or 2 days post mortem as a predictor of aged beef tenderness. Journal of Animal Science, v.75, p.2417-2422, 1997.

SLOSS, M.W.B.S.; KEMP, R.L.A.B. Veterinary clinical parasitology. 5.ed. Ames : Iowa State University, 1978. 247p.

TORRES, A.P.; JARDIM, W.R. Criação de cavalos e outros equiídeos. 3.ed. São Paulo : Nobel, 1985. 654p.

WEYNERMMAN, F.E.; DZAPO, V. Study of post mortem $\mathrm{pH}$ in horse. Fleischwirtschafl, v.77, n.2, p.1119-1121, 1997. 